\title{
Protein network involved in maintaining kidney permeability
}

A study published in the Journal of

Clinical Investigation reports on a protein network that has a key role in generating and maintaining the glomerular filtration barrier (GFB) in the kidney.

"Over the past few years an interesting concept has emerged regarding the similarities that govern podocyte organization and the mechanisms that mediate neuronal synapse development," explains researcher Shuta Ishibe. "For example, synaptopodin, an actinassociated protein expressed in synapses, is also expressed in podocytes where it plays an integral role in foot process stability. A role for dynamin in the maintenance of the GFB by modulating actin has also been suggested. Although synaptic vesicle recycling by clathrin-mediated endocytosis (CME) is a fundamental process in neurons, the importance of CME had not been investigated in podocytes. We therefore started evaluating key proteins involved in $\mathrm{CME}$ and how they regulate the GFB."

First, the researchers-in collaboration with Pietro De Camilli-showed that podocyte-selective knockout of the dynamin 1 and dynamin 2 genes in mice led to effacement of foot processes, severe proteinuria and kidney failure. Next, they demonstrated that knockout mice lacking either synaptojanin 1 or the three endophilin proteins (which are all key functional partners of dynamin at neuronal

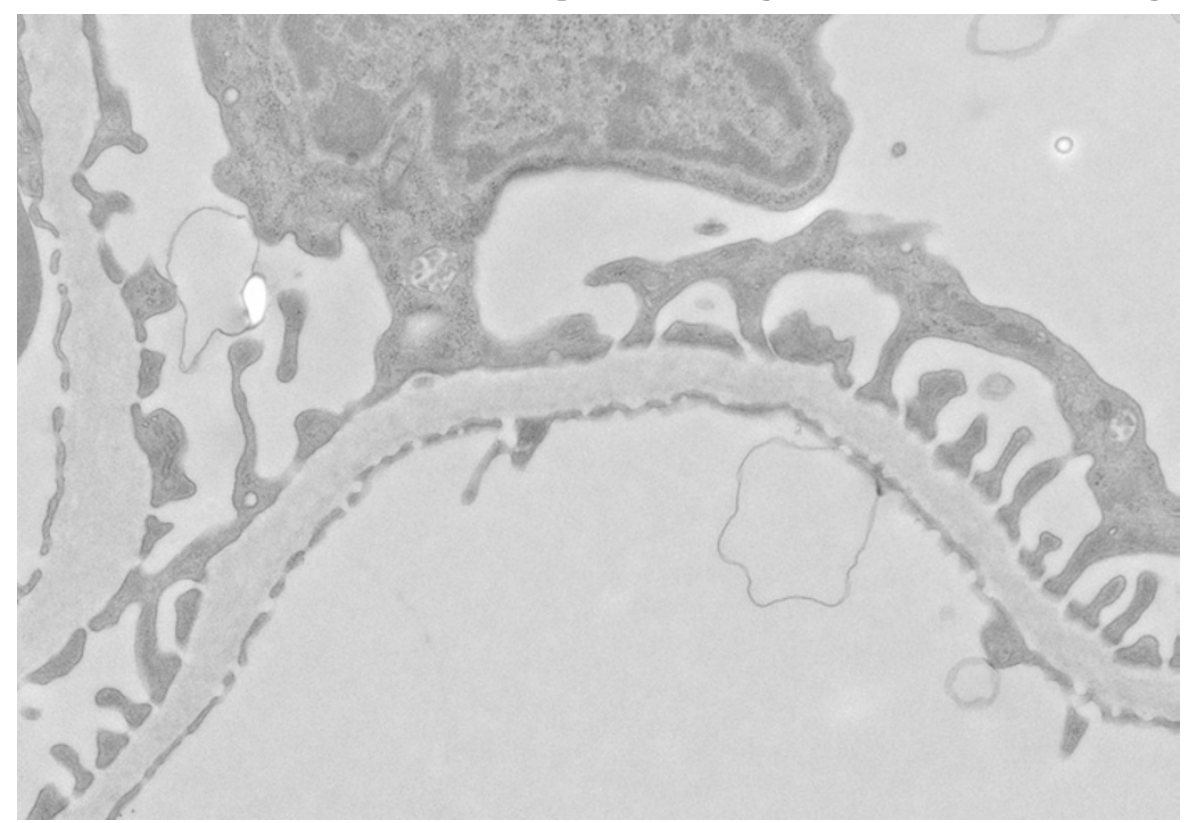

Electron micrograph showing normal podocyte foot process architecture in 8-week-old control mice. Courtesy of S. Ishibe, Yale University School of Medicine, New Haven, CT, USA.

synapses) were macroscopically normal at birth but had impaired neurological function and an underdeveloped GFB that resulted in severe proteinuria.

"In podocytes, we propose that synaptojanin 1, dynamin, and/or endophilin as well as their interacting proteins-CD2AP, Nck and Myo1E-play a key role in maintaining homeostasis in the GFB via interrelated functions in CME, signalling and actin dynamics," says Ishibe. "In the future, we would like to further investigate whether $\mathrm{CME}$ is regulating the internalization of extracellular hormones, signalling molecules, and/or transmembrane receptors involved in the development and maintenance of the GFB."

Rebecca Kelsey \author{
synaptojanin, and endophilin in podocyte foot processes. \\ J. Clin. Invest. doi:10.1172/JCI65289
}

Original article Soda, K. et al. Role of dynamin, 